\title{
LA MÉDIATION SOLITAIRE DE L'OMBUDSMAN DE PRESSE
}

\author{
Mário Mesquita ${ }^{1}$
}

La charge de médiateur dans la presse, créée aux États-Unis à la fin des années 60, n'appartient pas à la tradition européenne. Critiquer le journal au sein de ses propres pages correspond à une attitude contraire au courant traditionnel de la presse, les «règles de courtoisie» inhibant même -par exemple dans le cas des collaborateurs extérieurs- les remarques critiques. Les journalistes -au moins ceux de ma génération- ont appris dans les salles de rédaction, «sur le tas», à occulter erreurs et incorrections.

Le médiateur dans la presse quotidienne est une personnalité désignée par l'entreprise journalistique, en vue de recevoir les réclamations des lecteurs et de critiquer le quotidien. Il discute le journal dans ses propres pages, explique au lecteur des décisions éditoriales qui sont traditionnellement maintenues dans le secret des rédactions. Il possède un pouvoir d'influence et de parole. Il métacommunique,

1 Universidade Nova de Lisboa. Ancien directeur de la rédaction du Diário de Notícias et du Diário de Lisboa, il a été vice-président du Conseil de Presse (198485) portugais. Il a rempli la première charge de médiateur dans la presse généraliste portugaise (1997-98).

Recherches en communication, $\mathrm{n}^{\circ}$ 9, (1998). 
afin de légitimer et crédibiliser le journal par la réflexion sur sa propre pratique.

Il semble intéressant d'observer le rôle du médiateur dans la perspective de l'entreprise de presse, du discours journalistique et de l'autorégulation des médias. Sur le plan de l'entreprise, il représente l'institutionnalisation d'une critique régulière et d'un axe de liaison avec les lecteurs. Selon les cas, l'accent tonique sera mis sur le rôle de crédibilisation au moyen de la critique et de la correction d'erreurs, ou sur l'instrument stratégique et communicationnel en vue d'activer une certaine et, sans doute limitée, interactivité avec les destinataires.

Son action se déroule essentiellement a posteriori, ce qui garantit l'autonomie par rapport aux décisions éditoriales et évite également l'intromission dans la ligne hiérarchique de la rédaction. Cependant, le rôle de l'ombudsman ne se réalise pleinement que s'il y a un accord minimum sur les principes déontologiques applicables entre la hiérarchie interne, les journalistes et le médiateur. Si ce dénominateur commun n'existe pas, la répercussion de son travail dans la rédaction en subit naturellement les conséquences.

Sur le plan du discours, la voix du médiateur participe dans la construction de l'ethos du journal, autrement dit, il contribue à affirmer dans les textes sa crédibilité et son intégrité en tant qu'acteur de l'espace public. L'ombudsman participe à la fois au débat sur les médias, sur son propre quotidien et, simultanément, sur le sens et la définition de la charge qu'il exerce.

En instituant le médiateur, le journal se dote d'un «signe de qualité» ${ }^{1}$ face à ses lecteurs. C'est peut-être pour cette raison que la minorité qualifiée des journaux qui ont institutionnalisé le médiateur appartiennent à la catégorie des quotidiens de référence, puisque -comme l'écrit Hector Borrat- l'ethos «semble être la condition sine qua non de tous les journaux, mais il est possible que ce soit celui de type élitiste qui s'efforce le plus d'exhiber cette preuve $»^{2}$.

Sur le plan du système de la presse, le médiateur constitue une forme de régulation privée, entrepreneuriale et, dans la plupart des cas, professionnelle, étant donné que la charge a presque toujours été exercée par des journalistes ou des ex-journalistes. Dans une

1 J'emprunte l'expression à Jean Padioleau, mais en lui donnant une signification plus large (J. PAdIoleau, Le Monde et le Washington Post, Paris, PUF, 1985, p. 227).

2 H. BORRAT, El periódico, actor político, Barcelona, Gustavo Gilli SA, 1989, p. 108. 
classification très large, Claude-Jean Bernard inclut l'ombudsman de presse parmi «les moyens (non gouvernementaux) permettant d'assurer la responsabilité sociale des media», parallèlement aux organes collégiaux, comme les conseils de presse et les observatoires des médias, aux documents, comme les codes déontologiques, et aux processus, comme l'éducation et la formation professionnelle ${ }^{1}$.

Les entreprises veulent présenter les ombudsmen comme des outsiders, dans la mesure où le contrat qu'ils établissent avec le quotidien leur confère un certain degré d'autonomie. Mais le médiateur est choisi par la hiérarchie entrepreneuriale et rédactionnelle : «l'ombudsman est un insider, malgré les précautions souvent prises pour renforcer son indépendance» ${ }^{2}$. Parfois, il s'agit d'un journaliste (ou d'un ancien journaliste), ce profil ayant des inconvénients sur le plan de l'image, dans la mesure où il induit l'idée de complicité entre les pairs ou de partage d'une idéologie corporative d'autodéculpabilisation.

On soupçonne «la plupart» des ombudsmen de faire souvent déboucher les explications «sur quelque chose de ce type : nous faisons comme cela parce que c'est ainsi que nous le faisons ; c'est notre orientation» ${ }^{3}$. Un auteur comme Pierre Bourdieu, ne montre aucune empathie pour la figure du médiateur, qu'il considère comme «le défenseur de la ligne du journal» et qu'il associe à «la Nomenklatura des grands journalistes $»^{4}$.

Plus que le débat sur le statut ou le curriculum de celui qui exerce les fonctions, il convient de déterminer si l'ombudsman se limite à assurer la défense officieuse du journal face aux critiques provenant de l'extérieur ou si, au contraire, il se veut comme un critique interne, au nom d'un contrat implicite avec le lecteur.

L'ombudsman est une entité unipersonnelle, ce qui signifie que, selon le profil du titulaire de la charge, son exercice prend des caractéristiques différentes. Cette forte personnalisation traduit à la fois des

1 Cf. Cl.-J. Bertrand, La déontologie des médias, Paris, PUF, coll. "Que-sais-je?", 1997, p. 92 et passim.

2 Opinion de Robert J. Haiman («managing editor» du S. Petersburg Times). Cité par Edmond B. Lambeth, in Commited Journalism, Bloomington, Indiana University Press, 1986, p. 89.

3 C. TATE, «What do ombudsmen do ?», Columbia Journalism Review, mai/juin 1984. Cité à partir du «website» de l'Organization of the News Ombudsmen (http://www5.infi.net/ono/).

4 P. Bourdieu, Contre-feux, Paris, Liber-Raisons d'Agir, 1998, p. 87. Bourdieu se réfère en particulier au médiateur du Monde, Thomas Ferenczy. 
forces et des faiblesses. Elle permet la proximité par rapport aux journalistes, facilite la visibilité extérieure face aux lecteurs, mais elle lui retire, logiquement, le poids des décisions d'un organe collégial tel qu'un conseil de presse.

Certains auteurs soutiennent que l'ombudsman ne serait qu'une composante de «l'éthique stratégique», au service de l'entreprise journalistique. Même si cette analyse était, en partie, pertinente, il convient de rappeler que la définition de cette stratégie présuppose au moins la souplesse pour courir le risque d'une certaine polyphonie au journal.

\section{Un lecteur raisonnable}

Le médiateur construit, bien entendu, son lecteur-idéal. Il agit au nom d'un lecteur-citoyen, exigeant et intervenant. Le «reasonable reader» ${ }^{1}$-lecteur raisonnable et rationnel- auquel se réfèrent certains auteurs nord-américains, représente une construction composite, qui «personnifie l'idéal social d'un comportement raisonnable», de la part d'un consommateur d'informations ayant un certain degré d'exigences informatives.

L'action du médiateur ne concerne que les situations où le lecteur a été directement mis en cause. Les routines de la production journalistique -depuis les critères de sélection de l'information aux contraintes spatio-temporelles- portent parfois préjudice aux citoyens. Un cas intéressant s'est présenté au Diário de Notícias ${ }^{2}$. À l'occasion de la Journée de la Femme, le Président de la République portugaise a remis des décorations à plusieurs femmes portugaises. Les médias ont surtout retenu l'hommage rendu à des personnalités bien connues du «grand public». Pourtant, l'une des décorations les plus importantes et les plus anciennes -grand officier de l'ordre de Santiago da Espadaavait été attribuée à une personnalité du monde scientifique inconnue du «grand public», sur laquelle les médias ont maintenu un silence presque total.

Si une lettre n'avait pas été adressée au médiateur, les lecteurs du journal ignoreraient toujours le nom de celle qui est, peut-être, la

1 St. Klaidman et T. L. Beauchamp, The Virtuous Journalist, New York, Oxford University Press, 1987, p. 32 et passim.

2 M. MESQUITA, «A 'matemática' das condecorações», in $O$ Jornalismo en Análise, Coimbra, Minerva, 1998, p. 113. 
chercheuse portugaise dans le domaine des mathématiques la plus connue en dehors du Portugal. Le président a souhaité, par un geste symbolique, attirer l'attention du pays sur l'importance de la science et sur le rôle des scientifiques, mais les médias ont concentré leur attention sur les personnalités connues et sur les rivalités existant entre elles, accomplissant ainsi la tâche, qu'ils exercent si souvent, de réduire la politique au fait divers.

Cet épisode rappelle les théories d'Elisabeth Noëlle-Neumann ${ }^{1}$ sur la «spirale du silence» et la sensation d'impuissance que les citoyens ressentent lorsqu'ils sollicitent l'attention des médias sur un certain sujet, une certaine cause ou un certain principe, ou encore sur leur propre activité, et que les médias, sur la base de critères de sélection d'information, décident de ne pas leur prêter attention.

Les institutions, les entreprises, les personnalités publiques s'organisent en vue de faire pression sur les médias, mais les citoyens ordinaires sont simplement livrés à eux-mêmes. Les critères de sélection de l'information -même s'ils ne sont pas arbitraires- sont fragiles, subjectifs et discutables. On invoque l'actualité et on prépare des journaux télévisés de week-end, avec trois ou quatre jours d'avance. On en appelle à la nouveauté et on rend souvent hommage à la répétition et à l'homogénéisation de contenus entre les médias. On souligne le «human interest» et on oublie même des «récits de vie» et des parcours professionnels mal connus et ayant valeur d'exemple, tandis que l'on continue de graviter autour d'un cercle doré réduit, composé de personnalités dotées d'une mystérieuse propriété de médiatisation.

\section{Des personnes aux "sources organisées"}

Mais l'univers des destinataires du quotidien ne se limite pas à des personnes isolées qui se sentent offensées dans leurs droits ou qui manifestent le désir légitime de discuter leur journal. Il répond fréquemment à des plaintes et protestations provenant de puissantes organisations -le gouvernement, des entreprises, des institutions publiques ou privées- qui participent toutes au processus de construction des informations. Dans ce sens, l'ombudsman fonctionne comme

1 E. Nö̈lle-Neumann, La Espiral del Silencio - Opinión Publica: nuestra piel social, Barcelone, Paidós, 1995, p. 204 et passim. 
une instance de recours dans les «négociations» tourmentées et informelles entre journalistes et «sources».

L'intervention des «lecteurs-source» pose des problèmes particuliers. Au cours de mon expérience en tant que médiateur du quotidien Diário de Notícias, de Lisbonne, l'éditeur de la section de politique nationale m'a écrit afin de me signifier son désaccord à propos de certaines de mes prises de position, soutenant que la plupart des réclamations concernant son domaine «provenaient toujours de politiques», de telle façon qu'il ne savait pas si ces réclamations étaient «adressées au médiateur au service des lecteurs ou à un quelconque médiateur au service des acteurs (dans le sens d'intervenants de l'information)»1. Le journaliste suggérait que le «médiateur au service des lecteurs» -transformé en «médiateur au service des acteurs» ou des «sources»- pourrait objectivement être utilisé pour limiter l'indépendance des journalistes.

Bien que ces questions m'aient été posées dans un mémorandum interne, j'ai tenu à les clarifier publiquement, afin d'expliquer que, «la construction d'informations étant un processus de négociation, auquel participent sources et journalistes, il serait difficile de concevoir que le médiateur demeure tranquillement (ou qu'on le laisse demeurer) en marge de cette négociation permanente» ${ }^{2}$.

Il est naturel et même compréhensible que l'intervention du médiateur soit sollicitée par les «sources». Existe-t-il une possibilité d'instrumentalisation du médiateur par les «promoteurs des informations»? Sans doute. Mais son processus d'intervention est légitimé par une argumentation par laquelle il vise à persuader le lecteur. Les réclamations provenant de «sources» intéressées sont aussi légitimes que celles de tout autre lecteur. Les ignorer sous le prétexte qu'elles constituent des formes de pression sur le journal et les journalistes n'aurait donc aucun sens.

Au cours de mon expérience, j'ai répondu à des protestations provenant de «sources». Leurs réclamations étaient sans doute intéressées. Mais elles étaient souvent fondées, auquel cas elles ont reçu une réponse, ce qui a par exemple permis de corriger des situations où la rigueur de l'information était insuffisante ou d'irrespect du principe consistant à entendre les parties impliquées dans un conflit.

1 M. MESQUTTA, op. cit., p. 35.

2 Ibid. 
En interférant dans ce délicat domaine, l'ombudsman peut -en particulier s'il doit arbitrer, dans certains cas, en faveur des «sources»- susciter des protestations de la part des journalistes. Toutefois, celui qui accepte d'exercer ces fonctions sait bien qu'il ne se présente pas à un championnat de sympathie.

Contrairement à ce que l'on pourrait déduire de la phrase ironique sur le «médiateur au service des sources», le risque ne réside pas dans la relation entre le médiateur et les sources dûment identifiées, mais dans l'impossibilité de localiser et de comprendre pleinement les motivations de «promoteurs occultes» qui visent à influencer la construction d'informations. Sous cet aspect, l'invocation du devoir de secret de la part du journaliste -qui doit évidemment être respectée-place l'ombudsman et les lecteurs sur un pied d'égalité sur le plan cognitif, c'est-à-dire qu'ils sont réduits à la seule possibilité de formuler des conjectures.

\section{Le "journaliste de sources"}

Les «sources organisées» ne constituent que 1'un des «acteurs» en présence dans ce «champ du journalisme». Synthétisant diverses recherches dans le cadre de la théorie de l'information, C. Fagoaga explique que plusieurs chercheurs du courant du newsmaking «distinguent les journalistes organisés (professionnels territorialisés dans les médias) comme des acteurs importants, dans la mesure où ils interagissent avec des acteurs organisés (territorialisés dans les bureaucraties) pour partager mutuellement la production de l'information»' 1 .

Dans les conditions actuelles de production journalistique, la relation avec les sources est déterminante pour la propre carrière du journaliste. À ce propos, les analyses de Gaye Tuchman sur la relation entre l'accès du reporter à des sources haut placées et son prestige professionnel sont bien connues : «plus est élevé le status des sources et l'importance de leurs positions, plus est élevé le status du reporter» ${ }^{2}$.

\footnotetext{
1 C. FAgOAGA, «Fuentes Informativas», in A. BenITo (org.), Diccionario de Ciencias y Tecnicas de la Comunicacion, Madrid, Ediciones Paulinas, 1991, p. 607.

2 Cité par C. Fagagoa, op. cit, p. 609.
} 
Cependant, la question ne se résume pas à la vision simpliste et réductrice qui situe d'une part les journalistes et de l'autre les «sources». Souvent, au sein de la même organisation -par exemple au sein du gouvernement-certaines personnes ou certains départements luttent entre eux et cherchent à influencer les médias pour divulguer certains événements de la façon qui leur est le plus favorable ${ }^{1}$. Cette dispute pour l'accès aux médias favorise, à première vue, l'intervention du journaliste, dans la mesure où elle lui permet de confronter différentes stratégies en vue de définir l'information.

Les événements journalistiques peuvent être imprévus, mais ils sont fréquemment construits pièce par pièce par les stratèges de la communication politique, institutionnelle ou entrepreneuriale. Le puzzle informatif inclut, même lorsqu'il ne l'identifie pas, le travail des cabinets de communication ou des conseillers de presse. Mais tandis que ceux-ci valorisent l'information positive, destinée à construire l'image des institutions qu'ils représentent, les journalistes privilégient les événements qui possèdent les virtualités de déchaîner des conflits et d'activer l'intérêt des lecteurs.

Les critères professionnels de «mise-en-valeur» placent en général le reportage au sommet de la classification des formes d'expression journalistiques. Aucun autre «genre» ne s'adapte mieux à la définition du journaliste en tant que celui qui prête aux citoyens son œil d'observateur prétendument ingénu. De ce point de vue, le journalisme de reportage possède des virtualités supérieures au journalisme de sources, parce qu'il correspond à la vocation fondatrice de la profession et qu'il permet des recherches autonomes de l'intérêt stratégique de telle ou telle entité.

A ce propos, un journaliste du Diário de Notícias ${ }^{2}$ soutenait, dans une note adressée à l'ombudsman, que -dans la cotation au sein des rédactions- le modèle du «journaliste de sources» est plus valorisé que le «modèle du reporter» qui fonctionne sur la base de l'accès direct à l'événement : «(...) S'il est "seulement" un bon reporter, s'il est "seulement" doté d'une capacité particulière pour, chaque jour, prendre un cas, le suivre et le démêler, s'il possède "seulement" une excellente oreille de conversation, si "simplement" il raconte bien, avec aisance et imagination, les épisodes qu'il veut faire connaître, ce

1 Cf. R. SANTos, A negociação entre jornalistas e fontes, Coimbra, Minerva, 1998, p. 31. La référence de l'auteur vient à propos du commentaire à la notion de «premier définisseur», ainsi baptisé par Stuart Hall.

2 M. MEsquTTA, op. cit., p. 90-94. 
journaliste va ressentir combien il est difficile d'accompagner la carrière des "collègues qui ont des sources"».

Si les informations sont construites en négociation avec les sources, il est naturel que l'ombudsman s'insère également dans ce processus. Les médias et les acteurs politiques et sociaux interagissent dans le champ journalistique en vue de la production d'informations. Le médiateur introduit une nouvelle étape dans le processus de production journalistique, qui se caractérise par la modération d'un arbitre ne disposant que d'un pouvoir de parole.

\section{L'évaluation, une quatrième instance}

Harvey Molotch et Marilyn Lester ${ }^{1}$ ont identifié, dans une étude réputée des années 70 , trois instances principales dans la production de l'information: les promoteurs de l'information (personnalités, institutions ou cabinets), qui signalent ou créent un fait, les constructeurs de l'information (journalistes et éditeurs), qui transforment l'événement en information, et enfin les consommateurs de l'information (lecteurs ou spectateurs), qui reçoivent l'impact de l'information journalistique.

Le médiateur constitue une quatrième instance, appelée à intervenir a posteriori, à la demande des «consommateurs» ou des «promoteurs», en vue de réexaminer, du point de vue éthique, le processus d'information. On pourrait l'appeler évaluateur de l'information, dans la mesure où il peut rouvrir, en public, tout le processus d'élaboration de l'information, en favorisant le dialogue entre journalistes, source's et lecteurs.

L'affirmation des entreprises en tant qu'acteurs de l'espace public et la professionnalisation de la communication politique constituent -selon l'analyse de Gilles Achache ${ }^{2}$ - deux changements fondamentaux qui se sont opérés, au cours des années 80 . La communication politique, désormais professionnalisée, adopte «les pratiques du

1 H. MoLOTCH et M. LESTER, «As notícias como procedimento intencional : acerca do uso estratégico de acontecimentos de rotina, acidentes e escândalos», in N. TRAQUINA (org.), Jornalismo : Questöes, Teorias e «Estórias», Lisbonne, Vega, 1993, p. 34-51.

2 G. ACHACHE, «La communication : déclin ou extension de la politique ?», in Esprit, $\mathrm{n}^{\circ}$ 164, septembre 1990, p. 145-151. 
marché et de la compétition entre les acteurs économiques» ${ }^{1}$ qui leur étaient étrangères, tandis que les entreprises commencent à agir en se servant du discours politique comme modèle de référence.

Dans ce nouveau cadre, il est de plus en plus fréquent et délicat pour l'ombudsman de se voir confronté à des critiques provenant des secteurs de communication institutionnelle. Elles ne diminuent en rien le rôle du médiateur. Au contraire, elles élargissent son domaine d'intervention dans le processus de la production d'informations, lui permettant de soulever le voile qui, souvent, dissimule pudiquement le travail de «gatekeepers» anonymes et de sources sans visage, à l'intérieur et à l'extérieur des journaux.

L'intervention de l'ombudsman dans la relation complexe entre lecteurs, «sources» et journalistes est loin de correspondre aux mythologies de la protection des faibles contre les forts ou de quelqu'un qui serait situé «à mi-chemin entre Robin des Bois et Mère Teresa de Calcutta», comme l'a dit, avec une pointe d'ironie, l'écrivain Manuel Vasquéz Montalbán ${ }^{2}$. Mais l'activité du médiateur peut contribuer à rendre légitime l'intervention publique du journaliste, si l'on admet, avec Daniel Cornu, qu'il «ne tire sa légitimité que de la seule discussion (...) sur la vérité de son information et sur la manière dont cette information vraie rend compte des préoccupations réelles de la société» ${ }^{3}$.

L'ombudsman de presse ne doit être inclus parmi les candidats au titre de héros de la culture populaire comme, par exemple, le reporter d'investigation. Situé au centre d'un triangle infernal -lecteurs, sources et journalistes-, le rôle solitaire du médiateur risque toujours d'être mal compris et mal aimé. Mais il peut néanmoins développer la notion de responsabilité sociale de la presse. Et aider à réduire l'éloignement du lecteur face à son quotidien.

1 Ibid., p. 147.

2 Communication au congrès de la Organization of the News Ombudsmen (ONO), réalisé à Barcelona, les 5, 6 et 7 mai 1998.

3 D. CoRnu, Journalisme et vérité. Pour une éthique de l'information, Labor et Fides, Lausanne, 1994, p. 424. 